\title{
The effect of the volume of supra-inguinal injected solution on the spread of the injectate under the fascia iliaca: a preliminary study
}

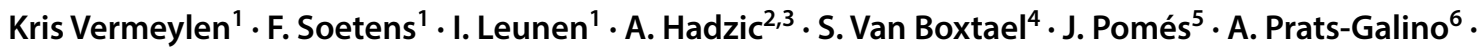 \\ M. Van de Velde ${ }^{7} \cdot$ A. Neyrinck ${ }^{7,8} \cdot$ X. Sala-Blanch ${ }^{9,10}$
}

Received: 16 May 2018 / Accepted: 14 September 2018

(c) Japanese Society of Anesthesiologists 2018

\begin{abstract}
The fascia iliaca compartment is the compartment confined by the fascia iliaca (FI) and a muscular layer formed by the iliac- and psoas muscle. This compartment creates a virtual tunnel that contains the femoral nerve (FN), the obturator nerve $(\mathrm{ON})$, and the lateral femoral cutaneous nerve (LFCN) of the lumbar plexus. In this pilot study, we aimed to determine the suggested volume needed to reach the three target nerves of the lumbar plexus (FN, ON, and LFCN) with a single-injection ultrasound-guided supra-inguinal fascia iliaca compartment (S-FICB). A computer tomography (CT scan)-guided step-up/step-down sequence was used to determine the suggested injection volume to target all three nerves. Subsequently, an anatomist blinded for the injected volume and CT findings, dissected the cadavers, and evaluated the spread of dye underneath the fascia iliaca. In total, seven pelvic areas of four cadavers were evaluated on CT scan and dissected. Distribution of dye underneath the FI in relation to the FN, ON, and the LFCN was recorded in all dissected cadavers. Combining CT and dissection findings, the suggested volume to reach the FN, ON, and LFCN with an S-FICB was $40 \mathrm{~mL}$.
\end{abstract}

Keywords Fascia iliaca $\cdot$ Fascia iliaca compartment block $\cdot$ Regional anesthesia $\cdot$ Anatomy of the hip $\cdot$ Anterior lumbar plexus block

Kris Vermeylen

Kris.vermeylen@gmail.com

1 Department of Anaesthesia, AZ Turnhout, Steenweg op Merksplas 44, 2300 Turnhout, Belgium

2 ZOL, Genk, Belgium

3 NYSORA, New York, NY 10025, USA

4 Department of Anaesthesia, ZOL, Genk, Belgium

5 Sección de radiología músculo-esquelética, Centro de Diagnóstico por la Imagen (CDI), Hospital Clínic, Universitat de Barcelona, Barcelona, Spain

6 Department of Anatomy, Laboratory of Surgical NeuroAnatomy (LSNA), Faculty of Medicine, Universitat de Barcelona, Barcelona, Spain

7 Department of Cardiovascular Sciences, KU Leuven, Leuven, Belgium

8 Department of Anaesthesiology, UZ Leuven, Katholieke Universiteit Leuven, Leuven, Belgium

9 Hospital Clinic, Universitat de Barcelona, Barcelona, Spain

10 Faculty of Medicine, Universitat de Barcelona, Barcelona, Spain
Regional anaesthesia has been proven to have a positive impact in the treatment of postoperative pain after hip surgery [1-4].

The fascia iliaca (FI) is the connective tissue layer on the surface of the iliac and psoas muscles. The virtual space between the FI and the muscles that the fascia covers forms the fascia iliaca compartment (FIC). The main nerves of the lumbar plexus, the femoral- $(\mathrm{FN})$, obturator- $(\mathrm{ON})$, and the lateral femoral cutaneous nerve (LFCN) are contained within the FIC as they travel on the iliacus muscle (IM) and more caudally between IM and psoas muscles (PM) [5].

The optimal injection volume of this approach to block the nerves of the lumbar plexus is yet to be determined.

The goal of this pilot study was to find the volume needed to reach the FN, the ON, and LFCN with a single-shot $\mathrm{S}$-FICB, using a step-up and step-down sequence in cadavers. A combination of computer tomography (CT) evaluation and anatomical dissection of the cadavers were performed to study the effect of different injection volumes. 
After approval by the Ethics and Research Committee of the University of Barcelona, 4 adult cadavers ( 2 men and 2 women; age between 68 and 86 years; weight between 55 and $72 \mathrm{~kg}$ ) with no history of spine or hip surgery were studied. The study was performed at the Department of Anatomy [laboratory of Surgical NeuroAnatomy (LSNA), Faculty of Medicine], University of Barcelona. The human cadavers were fresh frozen cadavers, frozen at $-20^{\circ} \mathrm{C}$, and defrosted $48 \mathrm{~h}$ prior to the research.

The mixture for injections was prepared with $80 \% \mathrm{NaCl}$ $0.9 \%$ mixed with $10 \%$ methylene blue (methylthioninium chloride Proveblue $5 \mathrm{mg} / \mathrm{mL}$-Martindale Pharma Essex, UK) to determine the spread of the injectate in the dissected human cadavers and 10\% iodine contrast (Iohexol 300, Omnipaque 300 - GE Healthcare) to detect the mixture on CT scan. The extent of spread of injected fluid after an S-FICB was examined at first by evaluation on CT scan and afterwards by anatomical dissection of the cadaver.
The CT scan evaluation was performed 30 min after injection and the spread of the contrast on this scan determined the injection volume of the next S-FICB.

The anatomical dissection evaluation of the spread (dye) was performed directly after the CT scan evaluation by an anatomist, blinded for the injected volume and CT scan findings.

The following algorithm determined the injection volume. Starting volume of the first S-FICB was $20 \mathrm{~mL}$. The volume of the next S-FICB was increased by $10 \mathrm{~mL}$ if not all three nerves (FN, ON, and LFCN) were reached on CT scan and decreased with $10 \mathrm{~mL}$ if all three nerves were reached with contrast. The $\mathrm{ON}$ is the most difficult nerve to reach because of its anatomical localization medially of the PM [6, 7]. Reachability of injectate towards the $\mathrm{ON}$ was considered positive if the contrast spread posteriorly and medially to the PM at any level between L4 and S1 on CT images, compatible with anatomical findings [6,7].

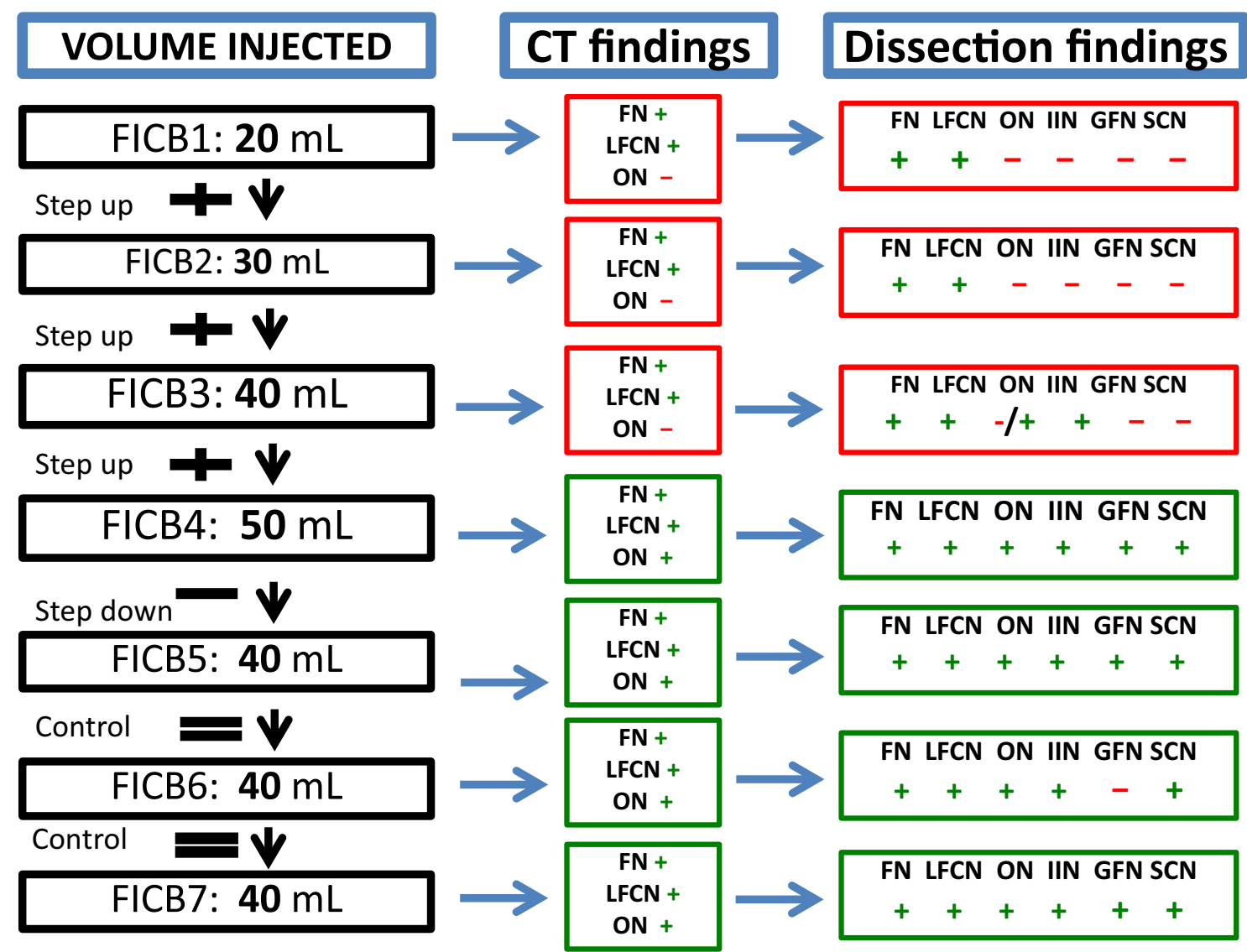

Fig. 1 Results of different volumes of injectate regarding the distribution towards the different nerves of the lumbar plexus, evaluation on CT scan imaging and dissection (S-FICB1-7). Cadaver 1: S-FICB 1 and 3; cadaver 2: S-FICB 2 and 4; cadaver 3: S-FICB 5 and 7; cadaver 4: S-FICB 6. $F N$ femoral nerve, $L F C N$ lateral femoral cutaneous nerve, $O N$ obturator nerve, IIN ilioinguinal nerve, GFN genitofemoral nerve, $S C N$ subcostal nerve. $(+)=$ positive for injectate reaching the nerve, $(-)=$ negative for injectate reaching the nerve 
This algorithm was followed until three consecutive S-FICBs reached the FN, ON, and LFCN at the volume tested. The methodology sequence is shown in the Fig. 1.

The target point of injection was underneath the FI and over the IM as described by Desmet et al. [8] [80-mm needle (22 G, Stimuplex Ultra 360; B. Braun Melsungen AG, Germany)]. If subfascial spread was visualized and dissection between the FI and the IM was not occurring, the injection was aborted and the needle was repositioned.

Once the needle in the correct position, the remaining volume was injected at a constant flow of $20 \mathrm{~mL} / \mathrm{min}$ using a pump (Alaris GH Syringe pump WD7INF023-Horwich UK). Injection of the mixture hydro-dissects the FI from the IM in a caudal to cranial direction.

The starting volume of the step-up/step-down sequence was set at $20 \mathrm{~mL}$ [9]. The same anaesthesiologist performed all S-FICBs.

Spread was evaluated using CT imaging and cadaver dissection.

CT scan images were obtained with a rotation time of $0.5 \mathrm{~s}$, and slice collimation of $0.6 \mathrm{~mm}, 120 \mathrm{kV}$, and $90 \mathrm{~mA}$ effective current. Axial, coronal, and sagittal reconstructions were performed with 3-mm sections (Somaton Sensation 64, Siemens Medical Systems, Erlangen, Germany). A radiologist specialized in musculoskeletal CT imaging and blinded to the injected volume, and determined the spread of the injected mixture in relation to the FN, ON, and the LFCN.

Anatomists, blinded for both the injected volume and the result of the distribution on CT imaging, dissected and assessed the spread of injectate in the anatomy laboratory of the University of Barcelona after the CT scan was performed.

After removal of the FI itself, the FN, ON, and LFCN were localized. The spread of the dye (methylene blue) was evaluated in relation to these three target nerves. Due to the medial and posterior anatomical localization of the $\mathrm{ON}$ in relationship to the PM, this muscle was opened to evaluate dye reaching the $\mathrm{ON}$.

In total, seven S-FICBs were performed on 4 cadavers. All cadavers were evaluated first by CT scan and afterwards by anatomical dissection.

Fig. 1 gives an overview of the CT findings regarding the spread of different volumes in correlation with the FN, $\mathrm{ON}$, and LFCN.

The $20 \mathrm{~mL}$ did reach the FN and the LFCN but not to the ON (S-FICB1), neither did $30 \mathrm{~mL}$ (S-FICB2). The spread of contrast around the $\mathrm{ON}$ in the third injection (S-FICB3) of $40 \mathrm{~mL}$ did reach the PM at the medial border but was evaluated as not reaching the $\mathrm{ON}$ by the radiologist, since the contrast did not reach the posterior border of the IPM on CT scan as per protocol. Following the study design, the next
S-FICB was performed with a $10 \mathrm{~mL}$ increment (volume of $50 \mathrm{~mL}-\mathrm{S}$-FICB4). The spread of this S-FICB reached all three target nerves. Following the algorithm, a decrease in injection volume of $10 \mathrm{~mL}$ ( $40 \mathrm{~mL}-\mathrm{S}$-FICB5) was used for the next injection. This $40 \mathrm{~mL}$ injection did, in contrast with the first $40 \mathrm{~mL}$ injection (S-FICB3), make contact with the $\mathrm{ON}$ on both the medial border a and the posterior border of the PM. After this successful $40 \mathrm{~mL}$ injection (all three nerves were reached by the contrast), two subsequent $40 \mathrm{~mL}$ injections (S-FICB6-7) were performed in order to meet the algorithm of three consecutive successful injections.

Post-acquisition 3D assessment of spread was obtained with DICOM images using MAC OsiriX software (OsiriX 8.0, Pixmeo Inc., Bernex Switzerland). OsiriX is an opensource software for navigating in multidimensional DICOM Images [5] (Fig. 2).

In total, seven pelvic areas of four cadavers (S-FICB1-7) were dissected. Distribution of dye underneath the FI in relation to the FN, ON, and the LFCN was seen in all dissected cadavers (Fig. 3).

Anatomical dissection could also evaluate dye (methylene blue) reaching other smaller nerves like the subcostal nerve (SCN), Ilioinguinal-iliohypogastric nerve (IIN), and genitofemoral nerve (GFN) (Fig. 1).

The anatomist did not see dye reaching the $\mathrm{ON}$ for the first two injections (S-FICB1-2). The first $40 \mathrm{~mL}$ (S-FICB3) showed a clear progressive and more substantial dye coverage of the psoas muscle and to the ON compared with the $30 \mathrm{~mL}$ injection (S-FICB2). For all consecutive injections (S-FICB4-7), the FN, ON, and LFCN were reached by the dye.

Concerning the smaller, not considered as target nerves, SCN, IIN, and GFN, the S-FICB3 did also reach the IIN. The dye of the S-FICB6 injection reached (together with the FN, ON, and LFCN) the SCN and the IIN. S-FICB5 and S-FICB7 showed a positive nerve evolvement for all the nerves (FN, ON, LFCN, IIN, SCN, and GFN) (Fig. 1).

In postoperative pain management after hip surgery, blocking the FN, LFCN, and ON with one injection is clinically relevant [10]. Before clinical relevance can be determined, the possibility to reach these three nerves with one injection needs to be demonstrated.

In this preliminary study, our data suggest that the spread of an injected volume after a S-FICB in fresh human cadavers is volume-dependent. CT scan evaluation and cadaver dissection determined that all three target nerves were reached in last three consecutive cadavers. The authors are aware of the fact that $10 \mathrm{~mL}$ is a large volume to be used as step-up/step-down sequence. However, this cadaver model must be seen as a pilot study to suggest a starting volume 


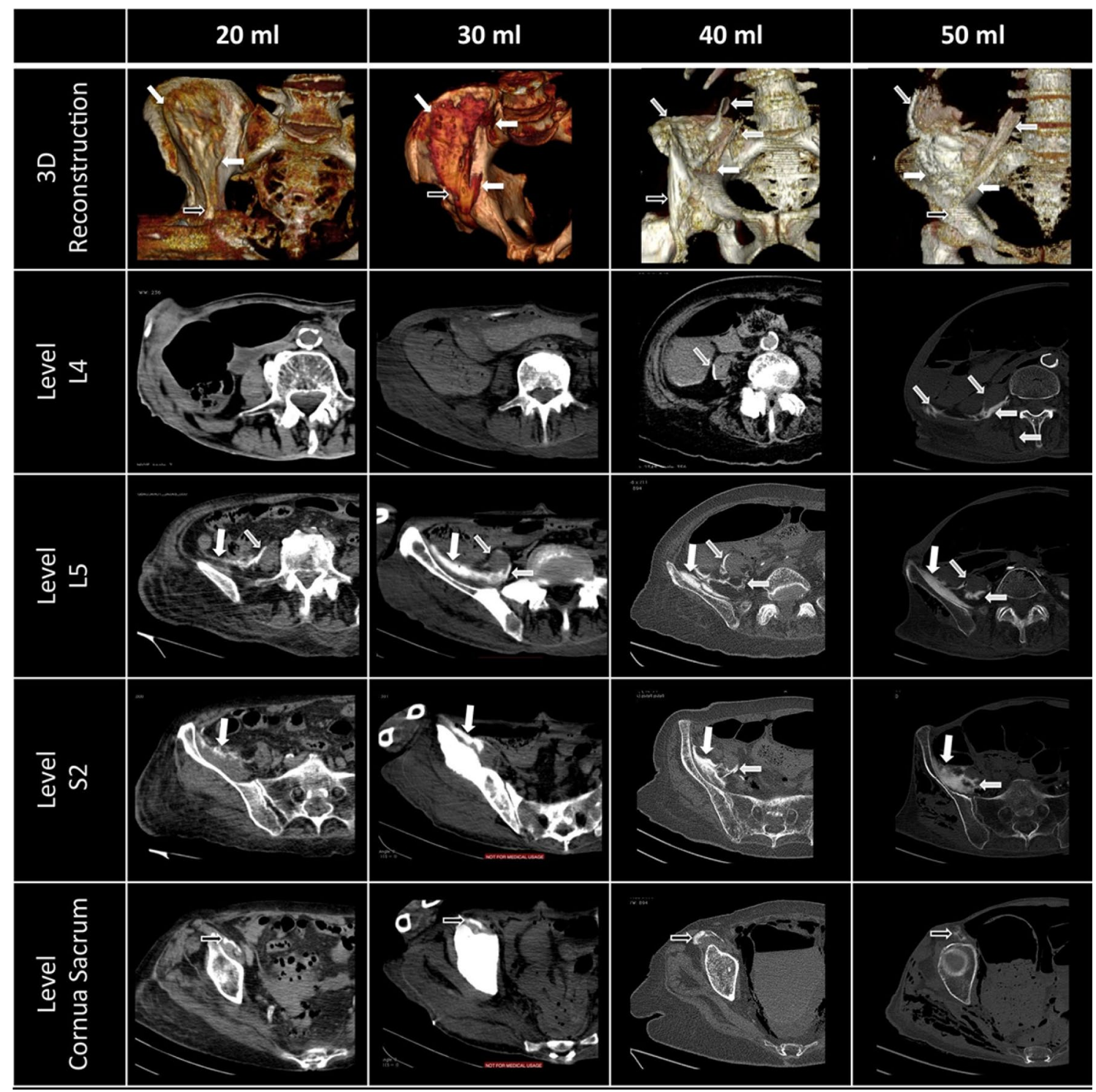

Fig. 2 CT scan cross sections and 3D reconstruction of increasing volumes (20-50 mL) at different levels (corpus vertebra L4, L5, S2, Cornua sacrum). With increasing volume there is a more cranial and medial distribution of the contrast. Arrows are pointing towards contrast

that at least is feasible in reaching all 3 target nerves: FN, $\mathrm{ON}$, and LFCN.
An important limitation of this study is the use of human cadavers. We chose to conduct our study on fresh, unpreserved specimens, because there is no literature on the effect of the preservation method in spread of fluids after injection. 
Fig. 3 Dissection of the pelvic region and the posterior abdominal wall (anterior-intra-abdominal view-40 mL-S-FICB 5).a Distribution of dye underneath the FI, reaching the different nerves:subcostal nerve (SCN), Ilioinguinal-iliohypogastric nerves (IIN), LFCN, FN, ON, genito-femoral nerve (anterior of the psoas) (GFN). b Note that the dye reaches the posterior part of the psoas muscle (PM) to stain the $\mathrm{ON}$. (PM* is $\mathrm{PM}$ after opening of the muscle or separating the superficial and the deeper part). c Anatomical section (S-FICB 5-40 mL) at the level just underneath the L5 corpus. Dye is distributed to the iliac and psoas muscle, staining the different nerves (arrows): obturator nerve (ON), lateral femoral cutaneous nerve (LFCN) and femoral nerve (FN); GFN genito-femoral nerve, $P M$ psoas muscle, $I M$ iliac muscle
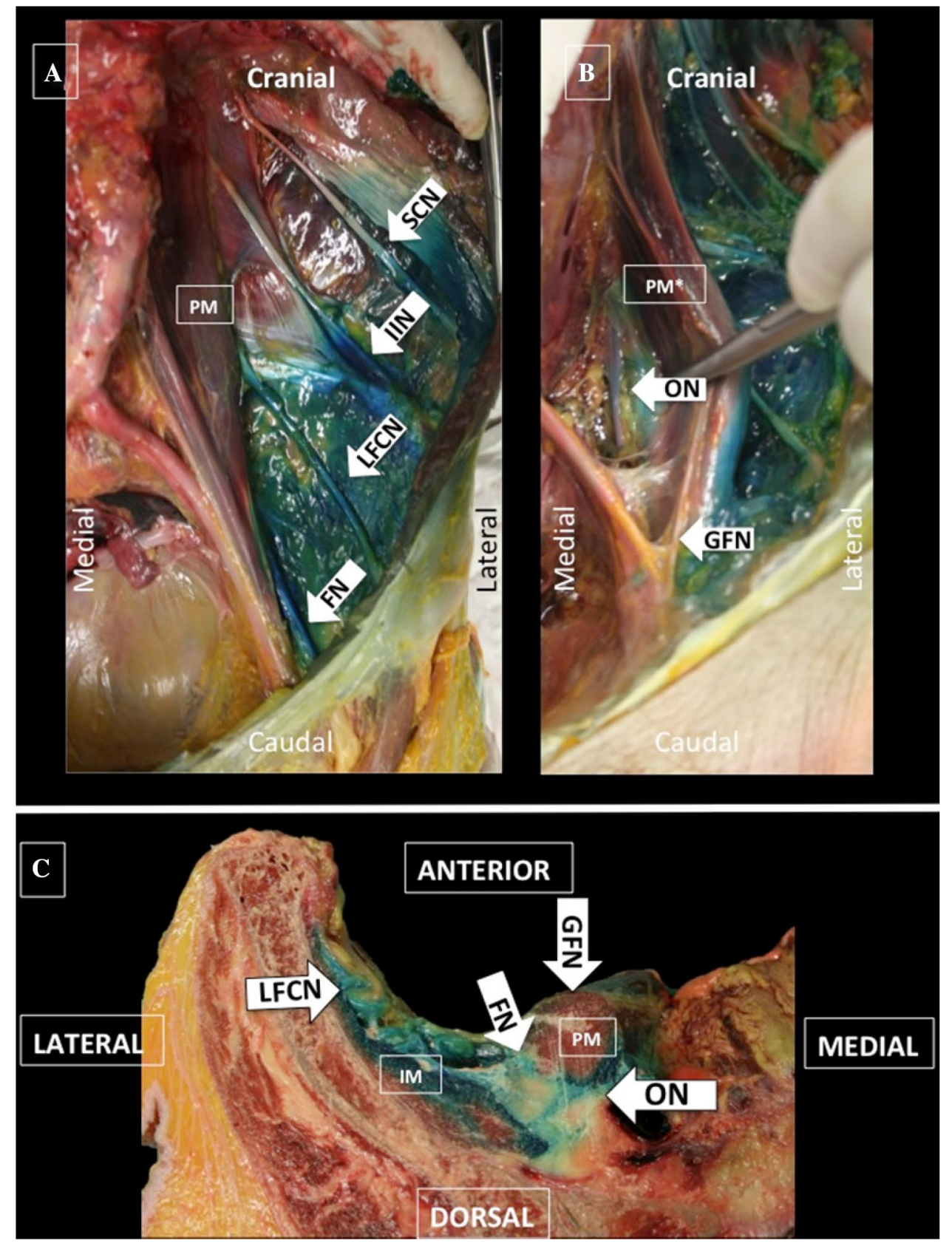

This human cadaver pilot study suggests that the volume of $40 \mathrm{~mL}$ is the best compromise among the volume choices that we used to reach the FN, ON, and LFCN in one time.

Author contributions KV: study design, study conduct, first draft of paper, and finalization of manuscript. XSB: study design and study conduct, literature research, data analysis and graphics, and critical appraisal of the final draft. IL: data analysis and critical appraisal of the final draft. FS: data analysis and critical appraisal of the final draft. MD, SVB, MVDV, and AH: critical appraisal of the final draft. JP: radiological support (CT scan), study conduct, and data analysis. AP-GG: anatomical support and study conduct.
Funding No additional funding.

\section{Compliance with ethical standards}

Conflict of interest No conflicts of interest.

\section{References}

1. Godoy Monzon D, Vazquez J, Jauregui JR, Iserson KV. Pain treatment in post-traumatic hip fracture in the elderly: regional 
block vs. systemic non-steroidal analgesics. Int J Emerg Med. 2010;3(4):321-5.

2. Hojer Karlsen AP, Geisler A, Petersen PL, Mathiesen O, Dahl JB. Postoperative pain treatment after total hip arthroplasty: a systematic review. Pain. 2015;156(1):8-30.

3. Husted H, Hansen HC, Holm G, Bach-Dal C, Rud K, Andersen KL, Kehlet $\mathrm{H}$. What determines length of stay after total hip and knee arthroplasty? A nationwide study in Denmark. Arch Orthop Trauma Surg. 2010;130(2):263-8.

4. Neuman MD, Silber JH, Elkassabany NM, Ludwig JM, Fleisher LA. Comparative effectiveness of regional versus general anesthesia for hip fracture surgery in adults. Anesthesiology. 2012;117(1):72-92.

5. Gerhardt M, Johnson K, Atkinson R, Snow B, Shaw C, Brown A, Vangsness CT Jr. Characterisation and classification of the neural anatomy in the human hip joint. Hip Int J Clin Exp Res Hip Pathol Ther. 2012;22(1):75-81.

6. Kirchmair L, Entner T, Wissel J, Moriggl B, Kapral S, Mitterschiffthaler G. A study of the paravertebral anatomy for ultrasound-guided posterior lumbar plexus block. Anesth Analg. 2001;93(2):477-81 (4th contents page).

7. Farny J, Drolet P, Girard M. Anatomy of the posterior approach to the lumbar plexus block. Can J Anaesth (Journal canadien d'anesthesie). 1994;41(6):480-5.

8. Desmet M, Vermeylen K, Van Herreweghe I, Carlier L, Soetens F, Lambrecht S, Croes K, Pottel H, Van de Velde M. A longitudinal supra-inguinal fascia iliaca compartment block reduces morphine consumption after total hip arthroplasty. Reg Anesth Pain Med. 2017;42(3):327-33.

9. Hebbard P, Ivanusic J, Sha S. Ultrasound-guided supra-inguinal fascia iliaca block: a cadaveric evaluation of a novel approach. Anaesthesia. 2011;66(4):300-5.

10. Memtsoudis SG, Poeran J, Cozowicz C, Zubizarreta N, Ozbek U, Mazumdar M. The impact of peripheral nerve blocks on perioperative outcome in hip and knee arthroplasty-a population-based study. Pain. 2016;157(10):2341-9. 